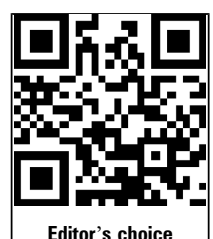

Editor's choice Scan to access mor free content

\title{
UK AMD EMR USERS GROUP REPORT V: benefits of initiating ranibizumab therapy for neovascular AMD in eyes with vision better than $6 / 12$
}

\begin{abstract}
Aaron Y Lee, ${ }^{1}$ Cecilia S Lee, ${ }^{2}$ Thomas Butt, ${ }^{3}$ Wen Xing, ${ }_{1}^{4}$ Robert L Johnston, ${ }^{5}$ Usha Chakravarthy, ${ }^{6}$ Catherine Egan, ${ }^{1}$ Toks Akerele, ${ }^{7}$ Martin McKibbin, ${ }^{8}$ Louise Downey, ${ }_{1}^{9}$ Salim Natha, ${ }^{10}$ Clare Bailey, ${ }_{1}^{11}$ Rehna Khan, ${ }^{12}$ Richard Antcliff, ${ }^{13}$ Atul Varma ${ }_{1}^{14}$ Vineeth Kumar, ${ }_{1}^{15}$ Marie Tsaloumas, ${ }^{16}$ Kaveri Mandal, ${ }_{17}^{17}$ Gerald Liew, ${ }_{1}^{18}$ Pearse A Keane, ${ }^{1}$ Dawn Sim, ${ }^{1,19}$ Catey Bunce, ${ }^{1}$ Adnan Tufail, ${ }^{1}$ on behalf of UK AMD EMR Users Group
\end{abstract}

For numbered affiliations see end of article.

\section{Correspondence to} Dr Adnan Tufail, Department of Medical Retina Moorfields Eye Hospital NHS Trust, 162 City Road, London EC1V 2PD, UK; Adnan.Tufail@moorfields.nhs.uk

AYL and CSL contributed equally and should be considered equivalent first authors.

Received 5 October 2014 Revised 11 December 2014 Accepted 12 January 2015 Published Online First 13 February 2015

\section{ABSTRACT}

Background/aims To study the effectiveness and clinical relevance of eyes treated with good (better than 6/12 or $>70$ Early Treatment Diabetic Retinopathy Study letters) visual acuity (VA) when initiating treatment with ranibizumab for neovascular age-related macular degeneration (nAMD) in the UK National Health Service. Currently eyes with VA better than $(>$ ) 6/12 are not routinely funded for therapy.

Methods Multicentre national nAMD database study on patients treated 3-5 years prior to the analysis. Anonymised structured data were collected from 14 centres. The primary outcome was the mean VA at year 1, 2 and 3. Secondary measures included the number of clinic visits and injections.

Results The study included 12951 treatment-naive eyes of 11135 patients receiving 92976 ranibizumab treatment episodes. A total of 754 patients had baseline VA better than 6/12 and at least 1-year of follow up. Mean VA of first treated eyes with baseline VA>6/12 at year $1,2,3$ were $6 / 10,6 / 12,6 / 15$, respectively and those with baseline VA 6/12 to >6/24 were 6/15, 6/17, $6 / 20$, respectively ( $p$ values $<0.001$ for comparing differences between $6 / 12$ and $6 / 12-6 / 24$ groups). For the second eyes with baseline $V A>6 / 12$, mean VA at year 1, 2, 3 were $6 / 9,6 / 9,6 / 10$ and those with baseline VA $6 / 12$ to $>6 / 24$ were $6 / 15,6 / 15,6 / 27$, respectively ( $p$ values $<0.001-0.005$ ). There was no significant difference in the average number of clinic visits or injections between those with VA better and worse than 6/12.

Conclusions All eyes with baseline VA>6/12 maintained better mean VA than the eyes with baseline VA $6 / 12$ to $>6 / 24$ at all time points for at least 2 years. The significantly better visual outcome in patients who were treated with good baseline VA has implications on future policy regarding the treatment criteria for $\mathrm{nAMD}$ patients' funding.

\section{CrossMark}

To cite: Lee $A Y$, Lee $C S$, Butt T, et al. Br J Ophthalmol 2015;99: 1045-1050.

\section{INTRODUCTION}

Age-related macular degeneration (AMD) is the leading cause of severe visual loss in patients over the age of 50 years in Europe and North America. ${ }^{1}{ }^{2}$ Neovascular AMD (nAMD) is characterised by choroidal neovascularisation (CNV), which is the growth of abnormal, choroidal blood vessels beneath the macula, which causes severe loss of vision and is responsible for the majority of visual loss due to $\mathrm{AMD}^{3}$ One of the key mediators implicated in the pathogenesis of CNV in nAMD is vascular endothelial growth factor-A (VEGF). Treatments for CNV (anti-VEGF agents) have high binding specificity for VEGF and are administered by repeated injection into the vitreous cavity. Intravitreal injection of anti-VEGF drugs such as ranibizumab is an established therapy to treat nAMD in the UK National Health Service (NHS). In the UK, the National Institute of Health and Care Excellence (NICE) approved the use of ranibizumab in August 2008, ${ }^{4}$ leading to almost exclusive usage of ranibizumab for nAMD in the UK NHS until the addition of aflibercept in 2013.

NICE, however, only recommended treatment with ranibizumab therapy if the visual acuity (VA) was in the range 6/12-6/96, consistent with the pivotal trials: Antibody for the Treatment of Predominantly Classic Choroidal Neovascularisation in Age-related Macular Degeneration (ANCHOR) and Minimally Classic/Occult Trial of the Anti-VEGF Antibody Ranibizumab in the Treatment of Neovascular Age-Related Macular Degeneration (MARINA) Studies. ${ }^{5} 6$ Our group has previously shown that if ranibizumab therapy is initiated at good $\mathrm{VA}$, the treated eye is more likely to maintain good vision. ${ }^{7}$ This is consistent with the indirect evidence from the pivotal trials that eyes are more likely to maintain vision than recover lost vision at the initiation of treatment. ${ }^{56}$

The proportion of eyes with nAMD detected with baseline vision better than $6 / 12$ has increased over time, due to increased awareness of the disease and surveillance of high-risk fellow eyes during the treatment of the first eye. ${ }^{8}$ Extrapolating from the earlier data, ${ }^{7}$ it seems reasonable to infer from these studies that treating at vision better that than $6 / 12$ is more likely to result in a patient remaining in the driving standard and maintain a better VA state. It is unlikely that a clinical trial would ever be conducted to replicate the ANCHOR and MARINA trial design for study eyes with better than $6 / 12$ vision at baseline, where the control arm gets deferred treatment, as equipoise does not exist in the treating community regarding 
the question- "is ranibizumab better than either no treatment or photodynamic therapy in patients with vision better than 6/12?" In this study, we have compared the visual outcome of patients receiving immediate treatment when vision was better than $6 / 12$ versus the patients who received treatment when the vision was $6 / 12$ or worse according to the NICE criteria. This study may help inform future policy decisions about whether to extend the funding of ranibizumab to vision of better than $6 / 12$ routinely.

\section{METHODS}

\section{Electronic medical record data source}

We have previously described the methodology of obtaining the large data set of 92976 ranibizumab injections. ${ }^{7}$ In brief, 14 NHS hospitals that deliver ranibizumab AMD treatment services in England and Northern Ireland submitted data to this study. Each site is the only NHS provider of nAMD care to their local population and very few patients switch between providers. Following NICE approval for the use of ranibizumab for nAMD in the NHS in August 2008 all sites used this drug almost exclusively during the study period. The lead clinician and Caldicott Guardian (who oversees data protection) at each centre gave written approval for the data extraction. Patient identifiers were completely stripped out and site and clinician data were pseudo-anonymised, and on this basis an ethics committee determined that formal ethics approval was not required. This study was conducted in accordance with the declaration of Helsinki and the UK's Data Protection Act.

\section{Data collection}

The study was initiated on 1 February 2012; Caldicott Guardian and lead clinician approval was achieved in 14 of the 18 contacted centres by March 2012, the date predetermined by the study team as the cut off before data extraction was to occur. All approvals and data extraction were performed by 2 April 2012. Data were delivered to the analysis team by the end of April 2012.

\section{Variables}

The electronic medical record (EMR) system used by centres in this study (Medisoft Ophthalmology, Medisoft Limited, Leeds, UK) has a structured data set for the management of nAMD that allows the rapid pooling of the data fields collected. This data set was defined and set up before the date of first data collection into this study. Data collected at all sites included VA for each eye (and the method of measurement) and treatment if required (with procedure details and complications). In many centres, the EMR system was used to collect a larger OCT data set including the presence or absence of parameters that influence re-treatment decisions.

\section{Data sources/measurements}

In this report, the 'best-measured VA' was the best VA with refraction or habitual correction and/or pinhole as measured on an Early Treatment Diabetic Retinopathy Study (ETDRS) chart and expressed as ETDRS letters. VA of 6/12 was defined by greater than 70 ETDRS letters. All analyses were performed using LogMAR VA.

\section{Statistical methods}

Data were extracted for both eyes of patients who had received at least one intravitreal injection of ranibizumab for nAMD. Both SPSS V.19 and R V.3.1.0 were used to combine, clean, reshape, merge, recode and analyse data. Continuous variables were analysed with a linear model and adjusted within the model with Tukey post hoc adjustment and among models with the Bonferonni correction. No imputation of missing data was undertaken.

\section{Missing data}

For patients where data were not available for a particular visit or had been lost to follow-up no missing value substitutions were performed. The only exception to this rule was baseline $\mathrm{VA}$ as some treatment centres brought patients back for a two-stop service: assessment on first visit followed by injection on second visit, and did not repeat VA measurements on the date of the first injection $(n=1670)$, which was always performed within 3 weeks. This was therefore not missing data per se but reflects variation in treatment delivery.

\section{RESULTS}

\section{Participants}

The 14 sites entered their first treatment episodes into the EMR system during the following years: 2006 ( $n=2$ sites), 2007 $(n=5), 2008(n=4), 2009(n=1)$ and $2010(n=2)$.

Anti-VEGF treatment was performed in 13774 patients and 2639 of them received bevacizumab for reasons other than nAMD. Thus, this study analyses data on 12951 eyes of 11135 patients who received a total of 92976 ranibizumab injections during 317371 clinic visits at 14 UK hospitals. $16.3 \%$ $(n=1816)$ of these patients required treatment to both eyes during the follow-up period. The demographics of the patients have been described previously. 89

All analyses were divided in the following three categories depending on unilateral or bilateral involvement: all, first and second treated eyes. All eyes were grouped in four different baseline VA categories: better than 6/12 (0.3 logMAR), 6/12 to better than $6 / 24(0.6 \log$ MAR), $6 / 24$ to better than $6 / 60$ (1.0 $\log$ MAR) and $6 / 60$ to better than $3 / 60$ (1.3 logMAR). These VA groups match four VA states groups used in the health economics analysis of the NICE submission for ranibizumab. ${ }^{4} 10$ One-year time period was defined by 12 months from the initial injection date for the specific eye.

\section{Number of patients}

Out of 11135 patients, 1816 of them had second eye involvement. During first year, there were a total of 4432 patients who underwent treatment for first eyes and 646 for the second eyes. During the second year, 1742 patients were treated for first eyes and 217 for second eyes. Four hundred seventy-seven patients were treated for first eyes and 47 for the second eyes during third year. As eyes were initiating treatment continuously during the period of study (2008-2012), there are far greater numbers of patients that have potential for 1 year follow up than 2 or 3 . Therefore, the reducing number per year reflects mainly the variable length of follow, rather than attrition. The actual loss to follow up rate was between $13 \%$ and $14 \% /$ year for the 2 years.

During the first year of treatment, there was a highly significant difference in starting VA between first and second eyes $(\mathrm{p}<0.0001)$. Mean VA in first eyes was $0.618 \log$ MAR $(6 / 25$ Snellen Equivalent) and $0.523 \log$ MAR (6/20) in second eyes. The distribution of the VA in first and second eyes was different. For first eyes, the VA group that had the highest number of eyes $(n=1664,36.9 \%)$ was the $6 / 12$ to $6 / 24$ category. This number was closely followed by $6 / 24$ to $6 / 60$ group ( $n=1654,36.7 \%$ ). In second eyes, the highest number of eyes was in $6 / 12$ to $6 / 24$ category $(n=273,41.7 \%)$ (figure 1$)$. 


\section{Number of visits}

The total number of clinic visits during 3 years did not vary significantly for either first or second eyes or when comparing eyes with baseline VA better than $6 / 12$ vs $6 / 12$ to $6 / 24$. For all eyes with baseline VA of better than $6 / 12$, the mean number of clinic visits during first, second and third year were 10.3, 8.5 8.6, respectively. The mean number of visits during first, second and third year were 10.2, 8.3, 8.4 in first eye group and 10.8, 9.5, 9.7 in second eye group. The mean number of visits in all eyes with baseline VA $6 / 12$ to $6 / 24$ during year $1,2,3$ were 10.2 , $8.2,8.2$, respectively. The mean number of visits for first and second eyes during year 1, 2, 3 were 10.1, 8.1, 8.2 and 10.5, 8.9, 8.1, respectively. For all (first, and second) eyes, there was no significant difference in the number of clinic visits during year 1,2 , and 3 between better than $6 / 12$ group vs $6 / 12$ to $6 / 24$
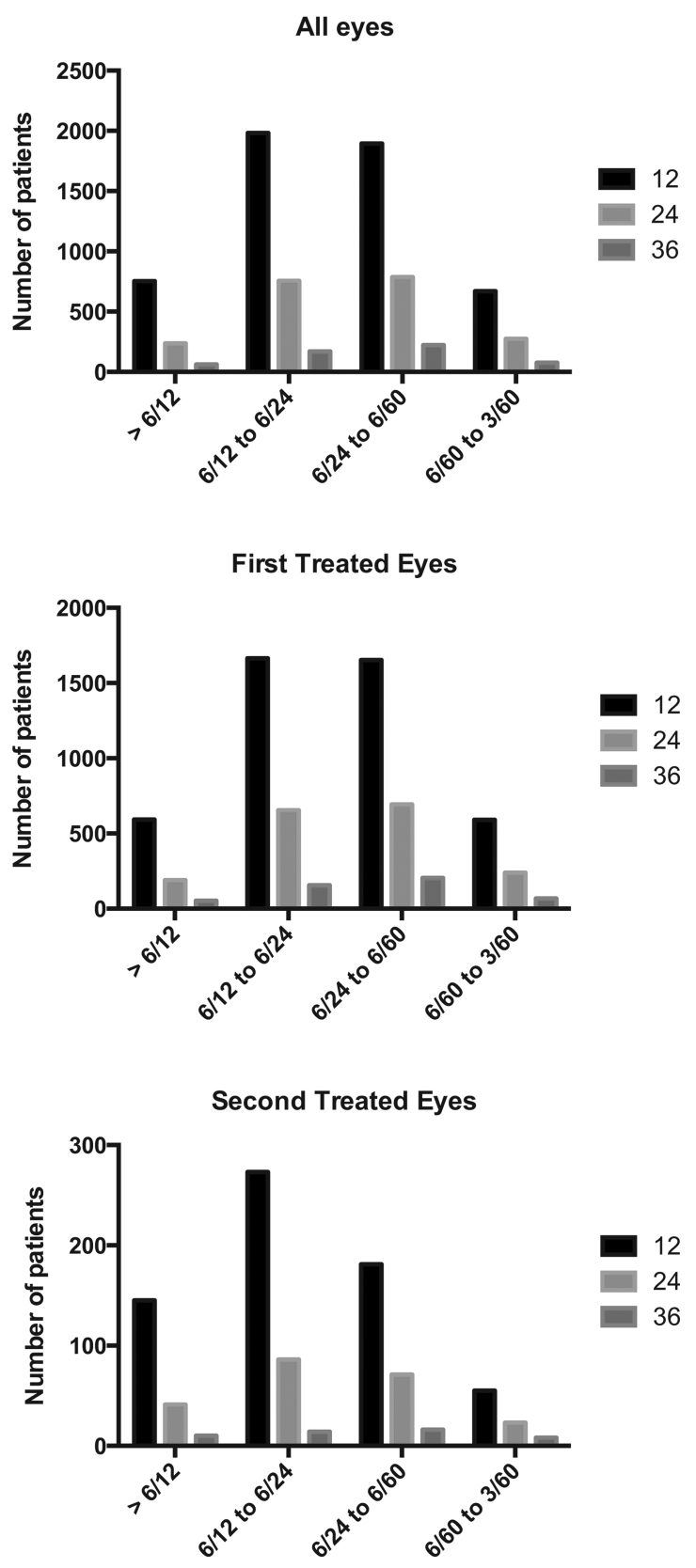

Figure 1 The number of eyes included in four baseline visual acuity groups, stratified by all eyes, first eyes and second eyes over a 3-year period. group (adjusted p values 0.13-0.98, 0.15-1.00, 0.99-1.00, respectively) (figure 2 ).

\section{Number of injections}

For all eyes with baseline VA better than 6/12, the mean number of injections during first, second and third year were 5.7, 2.3, 2.4 , respectively. The mean number of injections during first, second and third year were 5.7, 2.4, 2.6 in the first eye group and $5.5,1.9,1.8$ in the second eye group. For all eyes with baseline VA of $6 / 12$ to better than $6 / 24$, the number of injections during year $1,2,3$ were $5.9,2.7,2.8$, respectively. The mean number of injections during year 1, 2, 3 in this lower VA group for first and second eyes were 5.9, 2.7, 2.8 and 5.7, 2.7, 2.5, respectively. For all, first and second eyes, there was no significant difference in the number of injections at year 1,2 and 3 between better than $6 / 12$ group vs $6 / 12$ to better than $6 / 24$ (adjusted p values $0.15-0.60,0.11-0.95,0.63-1.00$, respectively) (figure 3).

\section{VA outcomes}

When all eyes were compared, the better the initial VA at time of treatment the better the VA at year 1, 2 and 3 (figure 4). Mean $\log$ MAR VA of first eyes with baseline VA better than $6 / 12$ at year $1,2,3$ were 0.223 (6/10 Snellen equivalent), 0.306 (6/12), 0.389 (6/15), respectively. Mean logMAR VA of the first eyes with baseline VA $6 / 12$ to better than $6 / 24$ at year $1,2,3$ were 0.408 (6/15), $0.464(6 / 17), 0.524(6 / 20)$, respectively. The difference in mean VA between baseline vision better than $6 / 12$ vs $6 / 12$ to $6 / 24$ was significant at year 1 and 2 but not year 3 (globally adjusted $\mathrm{p}$ values for year 1,2 , 3 were $<0.001$, $<0.001,0.40$, respectively). For the second eyes, mean logMAR VA with baseline VA better than 6/12 at year 1, 2, 3 were 0.176 (6/9), 0.197(6/9), 0.206(6/10), respectively. In contrast, the mean $\log$ MAR VA with baseline VA $6 / 12$ to $6 / 24$ at year $1,2,3$ were 0.385 (6/15), 0.401 (6/15), 0.647 (6/27), respectively. The difference in mean VA between the second eyes with baseline VA better than $6 / 12$ vs VA $6 / 12$ to $6 / 24$ was significant at all 3 years (globally adjusted $\mathrm{p}$ values for year $1,2,3$ were $<0.001$, 0.001 and 0.01 , respectively).

\section{DISCUSSION}

The prevalence of visual impairment due to AMD in UK has been estimated from $3.5 \%$ to $16 \%$ in those older than 75 or $77 .{ }^{11}$ It is assumed that approximately 400000 people are registered blind or partially sighted in Great Britain due to AMD. ${ }^{11}$ The visual impairment and need for continuous treatment associated with nAMD causes a significant burden at individual ${ }^{12} 13$ and societal level. ${ }^{14}$ Therefore, earlier treatment that leads to the maintenance of better visual function may be critical in reducing the visual impairment related to AMD.

To date, the focus on outcomes of treatment of nAMD has been on change in VA from baseline. However, change in VA alone is not a good indicator of patients' visual function and perception of their quality of life. ${ }^{13}$ Instead, the maintenance of a good functional visual state that allows continued reading and driving is of greater importance. Thus, rather than the absolute gain in VA, the duration that one can maintain good VA or reasonable visual function should be emphasised and taken into consideration when evaluating the benefits of any therapy for nAMD.

NICE currently does not recommend funding for eyes with good VA (better than 6/12), which may result in some patients having to wait for their vision to drop below $6 / 12$ to initiate therapy. The permitted VA for driving in UK is approximately $6 / 12$, thus the ability to achieve and maintain VA equal or 

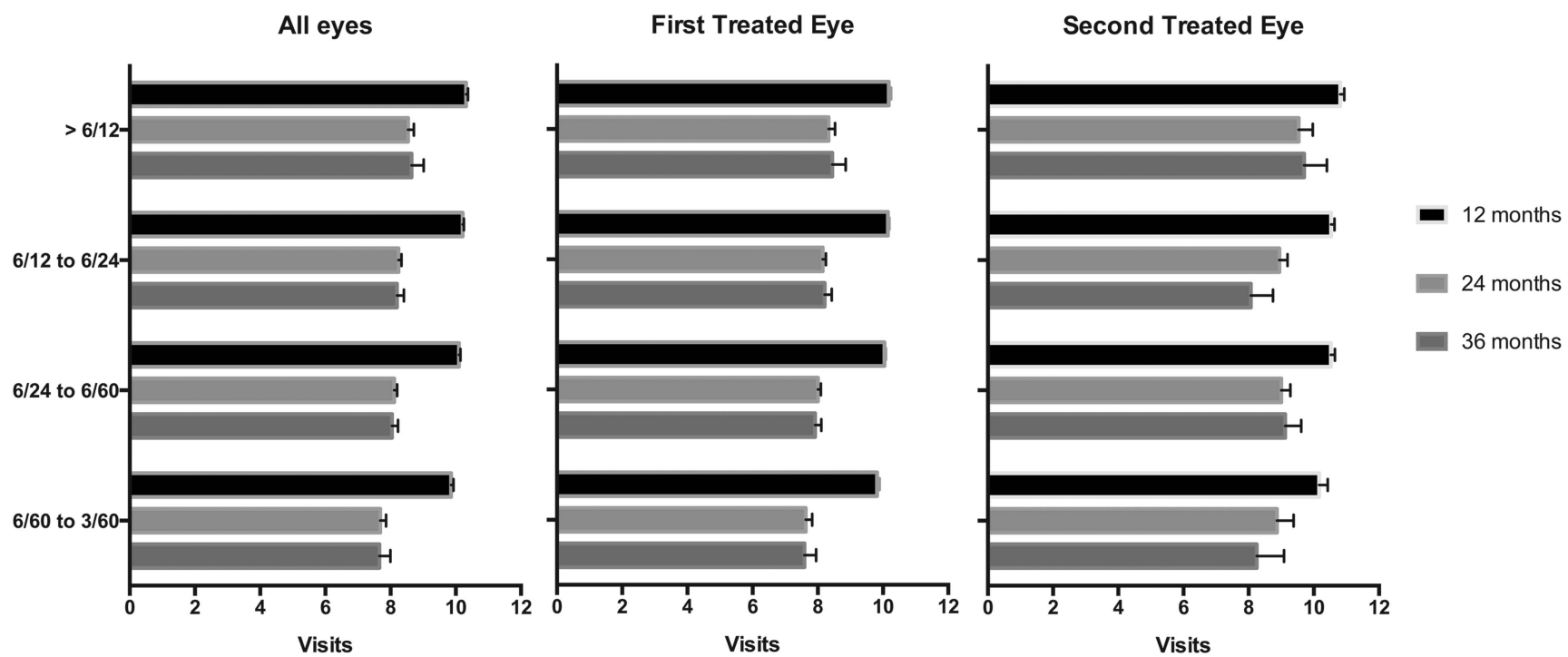

Figure 2 The number of clinic visits in four baseline visual acuity groups, stratified by all eyes, first eyes and second eyes over a 3-year period.

greater than $6 / 12$ is critical in many of our patients. This study shows the importance of earlier treatment stratified by baseline VA. We demonstrate that the earlier treatment group with baseline VA is better than $6 / 12$ is associated with better visual outcome and the longer maintenance of good VA without significant increase in the number of clinic visits or injections compared with the delayed treatment group.

\section{VA outcomes of eyes treated at better baseline visual} acuities

The overall VA outcome is better in eyes treated when baseline VA is better than $6 / 12$ compared with those with initial VA 6/12 to $6 / 24$. This difference is significant for 2 years when subgrouped into first eyes and for 3 years in second eyes. In addition, the second eyes tend to have better visual outcome than the first eyes as shown previously. ${ }^{8}$ The assumption is that there is a lower threshold to treat the second eye from the physician and the patient: the patient may monitor symptoms more regularly, and he or she may be under a closer follow-up regimen due to ongoing treatment of the first eye. Indeed, our data support better visual outcomes in second treated eyes. The difference in mean VA for baseline VA better than $6 / 12$ vs $6 / 12$ to $6 / 24$ was still significant at the end of 3 years for the second eyes. Thus, second eyes that are treated at better VA maintain better VA for longer periods than first eyes. Interestingly, the patients whose second eyes were treated when VA was better than 6/12 were the only group that maintained similar VA to their baseline VA at the end of 3-year period (figure 4). Even though the visual outcome of the eyes with baseline VA better than $6 / 12$ was better than the rest of the group, there was no difference in the number of visits or injections.

There are several studies supporting that visual outcome is better when treatment is initiated early. The time elapsed between the initial diagnosis of nAMD and treatment has been shown to correlate with the progression of visual loss. ${ }^{15}$ Weingessel et $a l^{16}$ reported worse VA at baseline, 6 and 12 months in patients with nAMD who had visual symptoms for longer period. Similarly, Canan et $a l^{17}$ demonstrated
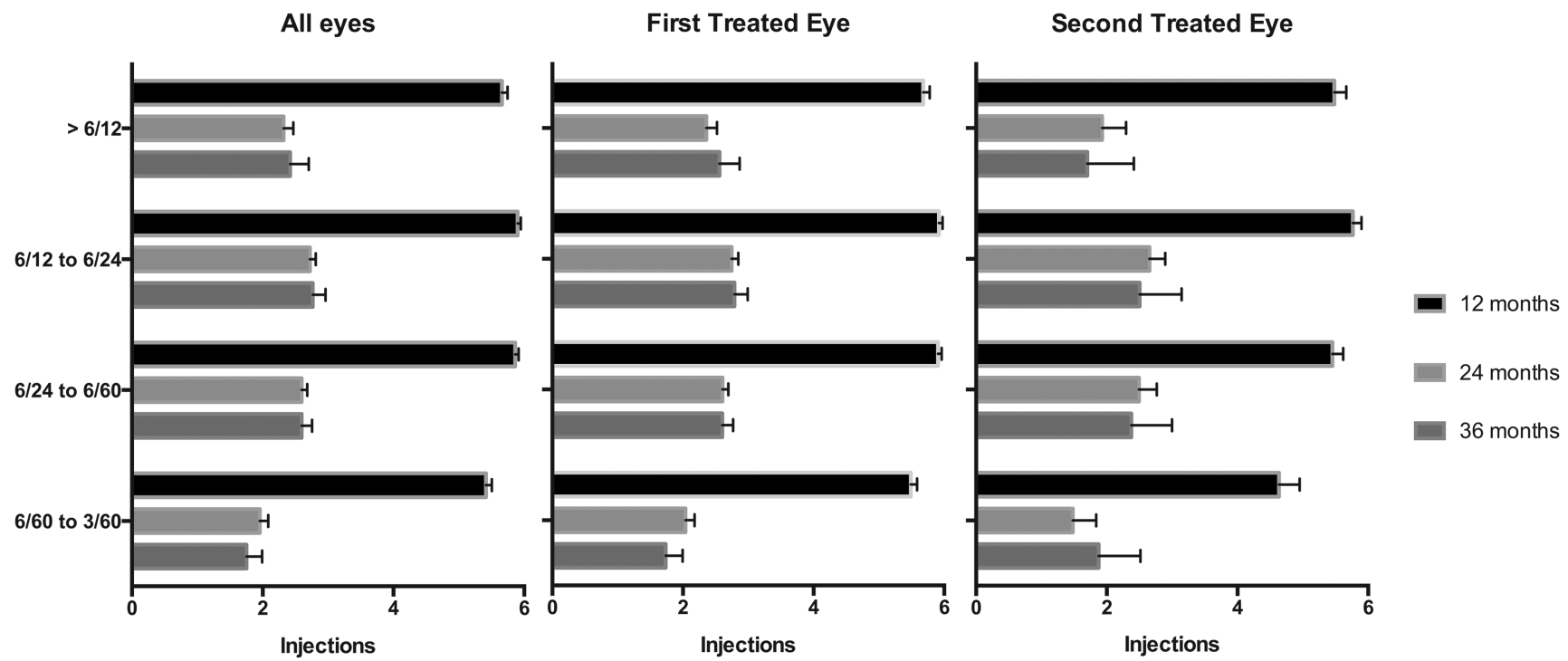

Figure 3 The number of injections in four baseline visual acuity groups, stratified by all eyes, first eyes and second eyes over a 3-year period. 
Figure 4 The mean visual acuity at baseline and year 1, 2, 3 in four initial visual acuity groups, stratified by all eyes, first eyes and second eyes.

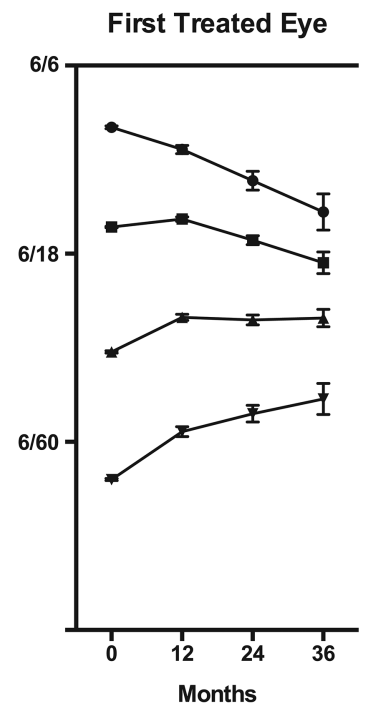

Months
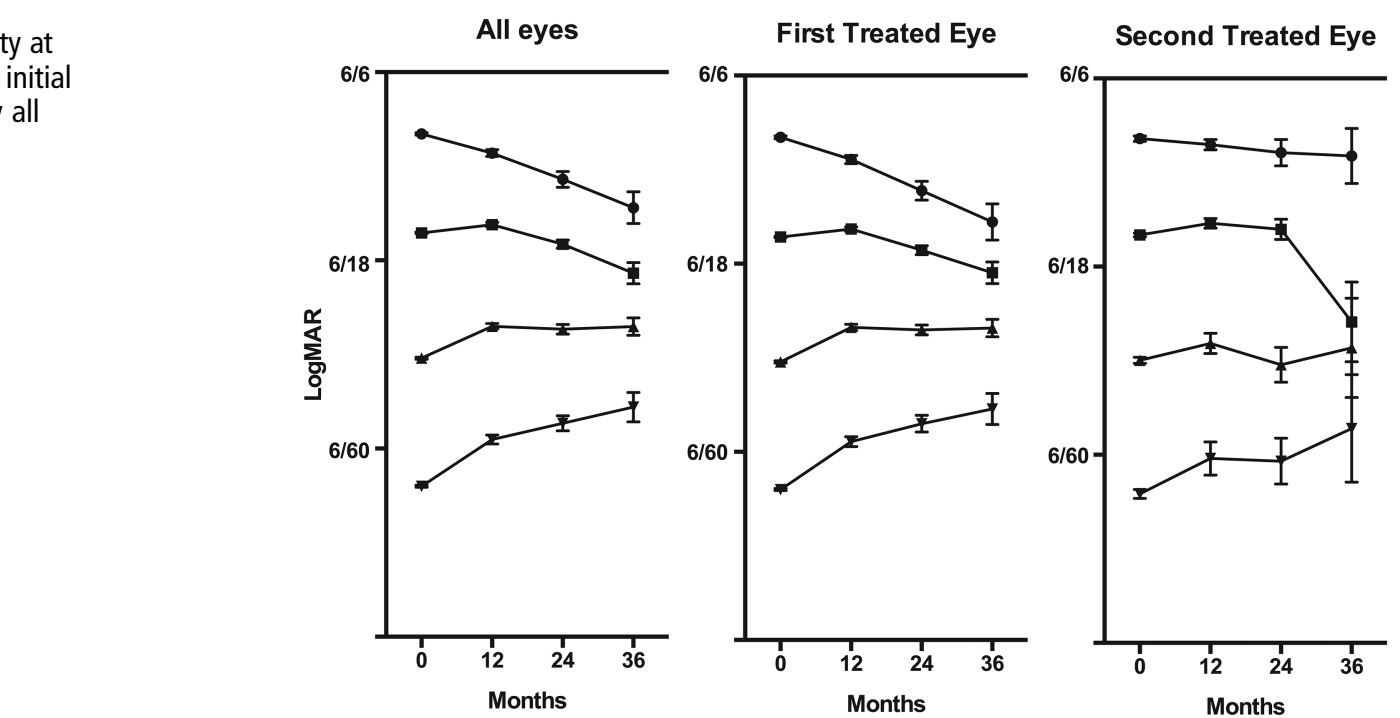

significantly better visual outcome in patients who had visual symptoms less than 1 month vs more than 1 month.

\section{Time to losing 6/12 vision}

The pivotal trials have shown that, over extended time periods, the VA and function of patients with nAMD continue to deteriorate despite regular treatment. ${ }^{18}$ Even though all patients continue to decrease with time in terms of their VA, the amount of time that they retain relatively good VA is critical for each patient. Figure 4 shows that the patients who started their treatment earlier when VA was better than $6 / 12$ retained better VA at year 1, 2 for first eyes and year 1, 2, 3 for second eyes when compared with worse baseline VA groups. However, the decreasing trend is similar to the rest of the group as expected from the results of trials. ${ }^{18}$

In two recent reports, Muether et al ${ }^{19} 20$ showed the outcome in a cohort of patients in Germany whose public health insurance allows ranibizumab only after reviewing each individual case. This approval process leads to a delay in treatment and their results showed significantly more distance ETDRS VA loss during the wait period than the letters gained during the consecutive therapy $(p=0.046)$. A similar trend was shown for near ETDRS VA but this was not significant $(p=0.219)$. In the UK, patients who do not meet the upper limit VA criteria may be obligated to wait and are therefore at risk for losing more VA during latency than what they will regain during consecutive treatment. The longer they wait with symptoms, the worse the visual outcome as shown in multiple reports. ${ }^{16} 17$

\section{Importance of early detection}

Our data emphasise the importance of early detection and frequent surveillance. Interestingly, the visual outcomes of the first eye being treated at VA better than $6 / 12$ vs the second eyes at better than $6 / 12$ vision are not the same. Despite similar initial VA at the time of initiation of therapy, the first eyes did worse than the second eyes at 3 years. Presumably, all patients treated for their first eyes had visual symptoms at presentation. Some delay between initial symptoms and subsequent first treatment is likely based on NHS referral patterns. In contrast, second eyes may be initiated on treatment due to OCT abnormalities that are noted during regular follow-up for patients' first eyes. They may or may not have been symptomatic at the time of treatment. Therefore, it is reasonable to infer that the second eyes are treated at an earlier disease state than the first eyes even if VA measurement cannot detect the difference.

Given the correlation of better visual outcome with earlier detection, closer surveillance methods should be explored in patients with nAMD. ${ }^{21}$ Ideally, these methods would allow frequent, easy monitoring at the patient's own home and at the same time be specific enough to identify nAMD progression. ${ }^{21} 22$

The main strength of this paper is the completeness of the data set by routine use of an EMR, which records all standardised data from each clinic visit. The data set represents real-world practice pattern and patients' outcome rather than from population studies or clinical trials. Nevertheless, limitations of this study are that it is retrospective in analysis (although the data is collected prospectively) and only includes UK hospitals that share the same EMR system. The results may not be as applicable in other countries that do not have similar treatment protocols or exclusion criteria. However, this provides real-world data that reflects daily clinical practice pattern rather than the protocols from the pivotal trials. There are few previously reported realworld data studies that provide insights into interpreting and incorporating the RCT data into daily clinical practice. ${ }^{23}$ In addition, this is the first study that demonstrates the visual outcome values in patients whose initial VA were excellent, given that these patients were excluded from most of the pivotal trials.

To our best knowledge, this is the first and largest study thus far reported in the literature to focus on the visual outcome of patients with baseline VA better than 6/12. This study supports the immediate treatment of nAMD when patients still maintain good vision. The cost-effectiveness of this early treatment to the healthcare system will be an important consideration and will be explored in a subsequent study. The study highlights important differences in the characteristics of the eyes with initial VA better than 6/12: significantly better mean VA compared with those with baseline VA $6 / 12$ to $6 / 24$ and the maintenance of this difference at 2 years for first eyes and 3 years for second eyes. This study may help inform future policy decision regarding the routine access to ranibizumab at visual acuities better than $6 / 12$.

\section{Author affiliations}

${ }^{1}$ Moorfields Eye Hospital NHS Foundation Trust, London, UK

${ }^{2}$ Department of Ophthalmology, University of Washington, Seattle, Washington, USA ${ }^{3}$ Institute of Ophthalmology, University College London, London, UK

${ }^{4}$ R\&D, Moorfields Eye Hospital NHSFT, London, UK 
${ }^{5}$ Gloucestershire Eye Department, Cheltenham General Hospital, Cheltenham, UK

${ }^{6}$ Belfast Health and Social Care Trust, Belfast, UK

${ }^{7}$ Hinchingbrooke Health Care NHS Trust, Cambridgeshire, UK

${ }^{8}$ Leeds Teaching Hospitals NHS Trust, Leeds, UK

${ }^{9}$ Hull and East Yorkshire Hospitals NHS Trust, Hull, UK

${ }^{10}$ Wrightington, Wigan and Leigh NHS Foundation Trust, Wigan, UK

${ }^{11}$ Bristol Eye Hospital, Bristol, UK

${ }^{12}$ Calderdale and Huddersfield NHS Foundation Trust, Huddersfield, UK

${ }^{13}$ Royal United Hospital Bath NHS Trust, Bath, UK

${ }^{14}$ Mid Yorkshire Hospitals NHS Trust, Yorkshire, UK

${ }^{15}$ Wirral University Teaching Hospital NHS Foundation Trust, Wirral, UK

${ }^{16}$ Department of Ophthalmology, University Hospitals Birmingham NHS Foundation

Trust, Birmingham, UK

${ }^{17}$ Department of Ophthalmology, Warrington and Halton Hospitals NHS Foundation Trust, Warrington, UK

${ }^{18}$ Centre for Vision Research, The University of Sydney, Sydney, New South Wales, Australia

${ }^{19}$ Department of Cell Biology, UCL Institute of Ophthalmology, London, UK

Collaborators Complete UK AMD EMR Users Group (listed alphabetically by center and within each center) Countess of Chester Hospital NHS Trust- Stewart Armstrong, Joey Cazabon Gloucestershire Hospitals NHS Foundation Trust-Quresh Mohamed, Ahmed Sallam, Javier Zarranz-Ventura Hull and East Yorkshire Hospitals NHS Trust-Seema Arora, Helen Cook, Kala Gopalakrishnan, Fiona Lyon, Tahir Islam, Naeem Zaman Leeds Teaching Hospitals NHS Trust—Oliver Backhouse, Tim Dabbs, Bryn Davies, Bataung Mokete, Damian O’Neill Moorfields Eye Hospital NHS Trust-Praveen J Patel.

Contributors All authors have given final approval of this version to be published. $\mathrm{RLJ}, \mathrm{CE}$ and AT participated in study codesigning, data collection and screening, data-analysis and evidence synthesis, and revising the manuscript. AYL, CSL and TB participated in literature search, data collection, data-analysis and evidence synthesis and drafting the manuscript. DS, PK and GL participated in hypothesis generation, evidence synthesis and revising the manuscript. WX, TA, MM, LD, SN, UC, CB, RK, $R A, A V, V K, M T, K M$ and $C B$ participated in study codesigning, data collection, screening and revising the manuscript.

Funding This work was supported in part by an unrestricted research award by Novartis Pharmaceuticals and NOTAL Vision. No member or affiliate of Novartis or Notal Vision had any input into data analysis, the interpretation of the data or writing the manuscript. This research has received a proportion of its funding from the Department of Health's NIHR Biomedical Research Centre for Ophthalmology at Moorfields Eye Hospital and UCL Institute of Ophthalmology. The views expressed in the publication are those of the authors and not necessarily those of the Department of Health.

Competing interests RLJ is the Medical Director of Medisoft Limited, the Electronic Medical Record software provider from which data were extracted.

Provenance and peer review Not commissioned; externally peer reviewed.

\section{REFERENCES}

1 Klein R, Peto T, Bird A, et al. The epidemiology of age-related macular degeneration. Am J Ophthalmol 2004;137:486-95.

2 Congdon N, O'Colmain B, Klaver CCW, et al. Eye Diseases Prevalence Research Group. Causes and prevalence of visual impairment among adults in the United States. Arch Ophthalmol 2004;122:477-85.

3 Ferris FL III, Fine SL, Hyman L. Age-related macular degeneration and blindness due to neovascular maculopathy. Arch Ophthalmol 1984;102:1640-2.

4 National Institute of Clinical Excellence (NICE) technology appraisal guidance 155. Ranibizumab and pegaptanib for the treatment of age-related macular degeneration. June, 2014. http://www.nice.org.uk/TA155
5 Brown DM, Kaiser PK, Michels M, et al.; ANCHOR Study Group. Ranibizumab versus verteporfin for neovascular age-related macular degeneration. N Eng/ J Med 2006;355:1432-44.

6 Rosenfeld PJ, Brown DM, Heier JS, et al.; MARINA StudyGroup. Ranibizumab for neovascular age-related macular degeneration. N Engl J Med 2006;355:1419-31.

7 Keenan TDL, Kelly SP, Sallam A, et al. Incidence and baseline clinical characteristics of treated neovascular age-related macular degeneration in a well-defined region of the UK. Br J Ophthalmol 2013:97:1168-72.

8 Zarranz-Ventura J, Liew G, Johnston RL, et al.; UK Age-related macular degeneration EMR Users Group. The neovascular age-related macular degeneration database: report 2: incidence, management and visual outcomes of second treated eyes. Ophthalmology 2014;121:1966-75. Article in press.

9 Keane PA, Patel PJ, Liakopoulos S, et al. Evaluation of age-related macular degeneration with optical coherence tomography. Surv Ophthalmol 2012;57:389-414.

10 Brown GC, Sharma S, Brown MM, et al. Utility values and age-related macular degeneration. Arch Ophthalmol 2000;118:47-51.

11 Owen $C G$, Fletcher $A E$, Donoghue $M$, et al. How big is the burden of visual loss caused by age related macular degeneration in the United Kingdom? $\mathrm{Br} J$ Ophthalmol 2003;87:312-17.

12 Dawson SR, Mallen CD, Gouldstone MB, et al. The prevalence of anxiety and depression in people with age-related macular degeneration: a systematic review of observational study data. BMC Ophthalmol 2014;14:78.

13 Slakter JS, Stur M. Quality of life in patients with age-related macular degeneration: impact of the condition and benefits of treatment. Surv Ophthalmol 2005;50:263-73.

14 Cruess AF, Zlateva $G, X u X$, et al. Economic burden of bilateral neovascular age-related macular degeneration: multi-country observational study. Pharmacoeconomics 2008;26:57-73

15 Oliver-Fernandez A, Bakal J, Segal S, et al. Progression of visual loss and time between initial assessment and treatment of wet age-related macular degeneration. Can J Ophthalmol 2005:40:313-19.

16 Weingessel B, Hintermayer G, Maca SM, et al. The significance of early treatment of exudative age-related macular degeneration: 12 months' results. Wien Klin Wochenschr 2012;124:750-5.

17 Canan H, Sizmaz S, Altan-Yaycioglu R, et al. Visual outcome of intravitreal ranibizumab for exudative age-related macular degeneration: timing and prognosis. Clin Interv Aging 2014;9:141-5.

18 Rofagha S, Bhisitkul RB, Boyer DS, et al.; SEVEN-UP Study Group. Seven-year outcomes in ranibizumab-treated patients in ANCHOR, MARINA, and HORIZON: a multicenter cohort study (SEVEN-UP). Ophthalmology 2013;120:2292-9.

19 Muether PS, Hermann MM, Koch K, et al. Delay between medical indication to anti-VEGF treatment in age-related macular degeneration can result in a loss of visual acuity. Graefes Arch Clin Exp Ophthalmol 2011;249:633-7.

20 Muether PS, Hoerster R, Hermann MM, et al. Long-term effects of ranibizumab treatment delay in neovascular age-related macular degeneration. Graefes Arch Clin Exp Ophthalmol 2013;251:453-8.

21 Chew EY, Clemons TE, Bressler SB, et al.; Appendix 1 for AREDS2-HOME Study Research Group. Randomized trial of the ForeseeHome monitoring device for early detection of neovascular age-related macular degeneration. The HOme Monitoring of the Eye (HOME) study design-HOME Study report number 1. Contemp Clin Trials 2014:37:294-300.

22 Group AHSR, Chew EY, Clemons TE, et al. Randomized trial of a home monitoring system for early detection of choroidal neovascularization home monitoring of the Eye (HOME) study. Ophthalmology 2014;121:535-44.

23 Rakic JM, Leys $\mathrm{A}$, Brie $\mathrm{H}$, et al. Real-world variability in ranibizumab treatment and associated clinical, quality of life, and safety outcomes over 24 months in patients with neovascular age-related macular degeneration: the HELIOS study. Clin Ophthalmol 2013;7:1849-58. 